\title{
INFLUENCIAS MORFOGENETICAS DE LOS DESALINEAMIENTOS Y LINEAS DE COSTA CONTRAPUESTAS EN EL LITORAL DE CHILE CENTRAL*
}

\author{
MORPHOGENETIC INFLUENCES OF OFFSETS AND \\ CONTRAPOSED COASTLINES IN THE CENTRAL CHILE SHORELINE*
}

\author{
JOSE F. ARAYA VERGARA \\ Departamento de Geografía Universidad de Chile
}

\begin{abstract}
Two morphostructural styles are present in the coastline Topocalma - Pichilemu $\left(34^{\circ}-34^{\circ} 30^{\prime} \mathrm{S}\right)$ : a) Offset style with zeta bays and b) Offset style with crenulate coastlines and almost straight beaches. They are resulting from contraposed shorelines, as evidenced by overlapping softer desposits on crystalline hard rocks.
\end{abstract}

System atributes are recognized in these styles by using components as offset angle, asymetry, orientation and curvature index. Correlation and matrices analysis indicate that 40 $60 \%$ of the effects are explained by three components: offset angle, curvature index and azimuth. Therefore, both of the styles may be recognized as subsystems.

Each subsystem presents a different behaviour in front of wave environment. Since offset condition and orientation explain curvature, each style receives differential wave attack, because refraction is well and positively correlated to curvature.

Half heart shaped beaches and bays represent, therefore, both drift and swash alignment, depending on the offset angle and refraction of constructing waves. So, a logarithmic spiral may appear. Their maximum concavity is eroded during surges, meanwhile the downdrift part can suffer depositional effect. This difference is not so clear in the almost rectilinear beaches of the other style. State and attack conditions depend, therefore, on the position in the bay. Dune system structures associated to beaches may be correlated to sedimentary budget and beach orientation.

This fact must be taken into account in decision making processes concerning shore management.

Key words and ideas: offsett, zeta from bays, logarithmic spiral, crenulated shorelines, contraposed shorelines, attack condition, state condition.

Palabras e ideas claves: desalineamiento, bahias en zeta, espiral logaritmica, lineas litorales dentadas, lineas litorales contrapuestas, condición de ataque, condición de estado.

"Trabajo realizado dentro del proyecto "Sectores de progradación y de erosión en Chile Central", auspiciado por el Departamento de Desarrollo de la Investigación de la Universidad de Chile. 


\section{INTRODUCCION}

\subsection{ANTECEDENTES}

En el análisis preliminar de la localización de los procesos y formas predominantes de la linea litoral de Chile (ARAYA-VERGARA, 1982a) fue reconocida una región de acantilados blandos regularizados alternados con "bluffs" duros y "pocket beaches", activados por "swell" de S.W.e "inputs" fluviales periódicos mayores, entre Punta Curaumilla y Dichato (Chile Central). Los sectores de acantilados regularizados son tres, elaborados en areniscas y lutitas relativamente blandas del Terciario Superior. Del mapa publicado en esa oportunidad, se desprende que entre los tres sectores se ubican dos tipos de línea litoral: arenosa regular y rocosa irregular. Este último es el que contiene la alternancia entre "bluffs" duros y "pocket beaches". Posteriores revisiones de campo permitieron comprender su importancia en el programa general de investigación.

\subsection{OBJETIVOS}

El programa sobre "determinación de sitios costeros de interés geomorfológico, etc." en su fase 3, "Sectores de progradación y de erosión en Chile Central", pretende llegar a la noción de estado, distinguiéndose entre avance, retroceso y estabilidad de diferentes tipos de línea litoral. Para ello, es necesario determinar la condición de ataque y la condición de estado, expresiones que serán definidas más adelante. Las últimas experiencias de terreno demostraron que ambas condiciones pueden variar según el tipo de sector. Los sectores de acantilados regularizados presentan condiciones de ataque $y$ de estado más independientes de las refracciones del oleaje que los sectores intermedios rocosos e irregulares. Mediante una primera aproximación, hay razones para hipotetizar que mientras los sectores de acantilados regularizados presentan una estabilidad de mediano término, las salientes cristalinas de los sectores intermedios tienen estabilidad de largo término, causa esencial de irregularidades.

Revisando la linea de costa desde Punta Barranco $\left(34^{\circ} 02^{\prime}\right)$ hasta Cáhuil $\left(34^{\circ} 30^{\prime}\right)$, se encontró que la condición de ataque y la condición de estado de las playas varian según la posición de éstas en la ensenada, de modo que es difícil evaluar su estado sin tener en cuenta patrones morfoestructurales y evolutivos más generales. Por lo tanto, las metas del presente trabajo consisten en: 1) precisar las morfoestructuras fundamentales que tienen que ver con la irregularidad; 2) señalar hipótesis adecuadas sobre la evolución general; 3) determinar la influencia de estos factores en características morfométricas de la línea litoral (como la asimetría de bahías, orientación, e indice de curvatural, y 4) señalar las posibles relaciones de estos hechos con el grado de estabilidad actual.

Analizando los cambios recientes en las líneas litorales del mundo, BIRD (1981:26) señala que "la tarea de ordenar los factores relevantes - prorrateando su contribución a los cambios que han ocurrido en sectores particulares - requiere mayor investigación, tanto de patrones y montos de cambio como de sistemas de procesos que operan en las aguas costeras".

\subsection{METODO Y TECNICA}

\subsubsection{El problema}

La primera fase de observación consistió en determinar en el terreno las condiciones de ataque y de estado de la línea litoral. Se entiende por condición de ataque el conjunto de requerimientos necesarios para que una forma determinada de la linea litoral sea atacada por el oleaje. Se trata entonces de un umbral témporo-espacial. Denominase condición de estado (Zustand) el conjunto de factores que permiten la permanencia de una forma determinada de la línea litoral. Se trata, pues, de falta de umbral. Los elementos para determinar estas propiedades se refirieron sólo al perfil transversal a la línea litoral (shoreline), pero no a las influencias de la configuración en planta. La perspectiva de buscar otros elementos de análisis surgió cuando se advirtió en terreno que para puntos muy cercanos de una misma bahia los perfiles mostraban procesos diferentes y a veces antinómicos en la playa. Al seleccionar los lugares de observación en las fotos aéreas, esta diferencia no habia podido percibirse. Al encontrarla en terreno, fue asociada sobre todo a las bahias en forma de zeta griega entre Punta Barranco y Cáhuil que se alternan con sectores dentados y desalineados (Fig. 1). Había que definir estos factores de la dinámica actual, lo que llevó a observar con mayor detención los acantilados inmediatos y puntas rocosas en cuanto a la 




Fig. $1 \mathrm{~A}$ 


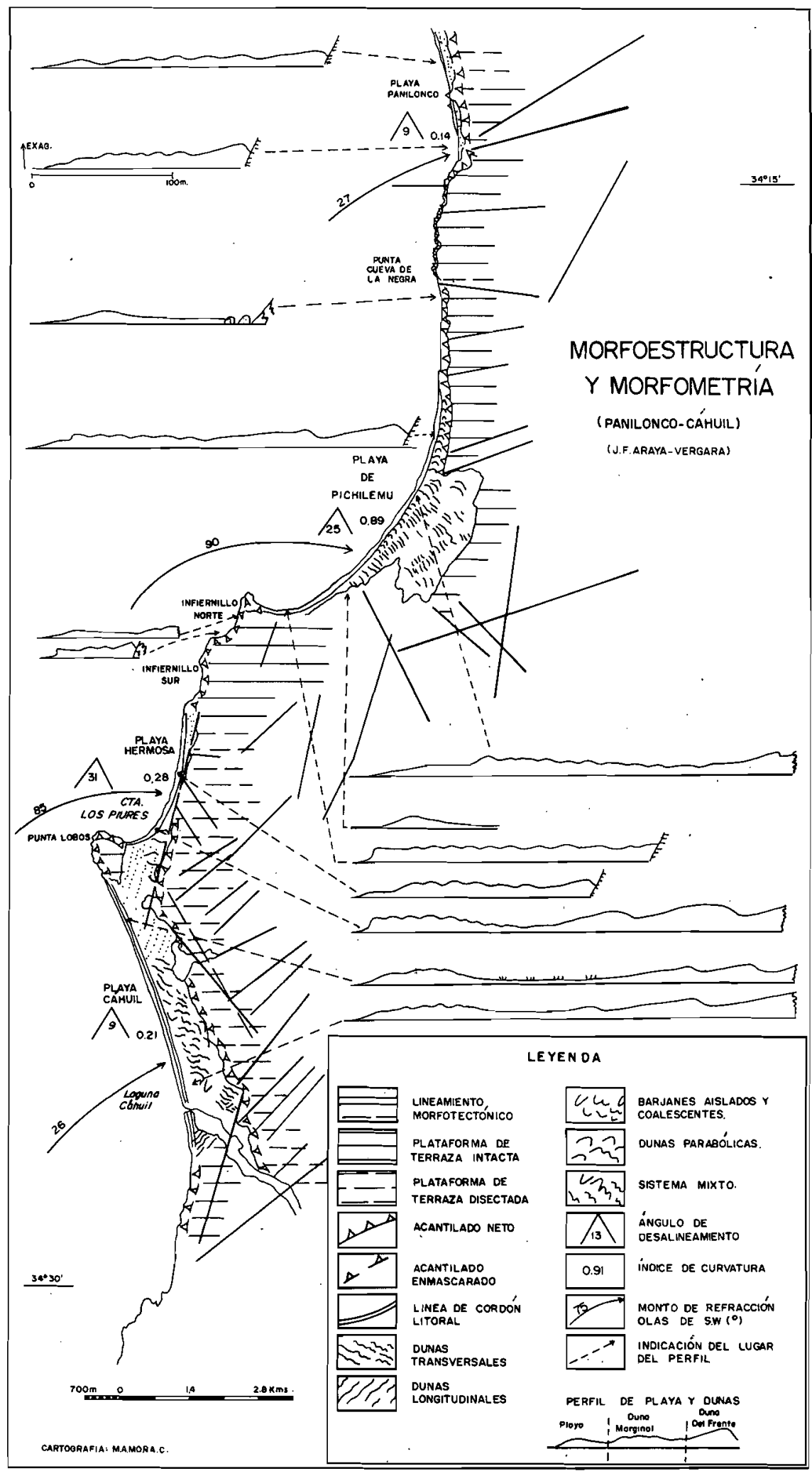

Fig. 1 B 
litología, estratigrafía de su cuerpo y características tectónico fracturales de las rocas cristalinas del zócalo. Ello condujo al descubrimiento de estructuras discordantes y desalineamientos morfotectónicos, hecho resultante del efecto de "serendipity" -o buena suerte para encontrar un fenómeno importante cuando se busca otro- concepto que debiera emplearse en el lenguaje sobre metodología de la ciencia, ya que permite ampliar los horizontes paradigmáticos.

\subsubsection{Naturaleza de la hipótesis}

A partir del efecto de "serendipity" se puede seguir un camino deductivo-hipotético. Es posible empezar comparando las condiciones de la línea de costa irregular con las de la línea de costa regular, incluyendo el comienzo de la costa "sensu stricto", en perfil transversal. Así, mientras los sectores al Norte y al Sur del estudiado presentan una línea de costa acantilada en areniscas $y$ regular, este último muestra el acantilado en areniscas sólo en las partes altas de acantilados abandonados frente a las entrantes de bahías o playas de bolsillo y a veces frente a las puntas (Fig. 2). Estas últimas siempre corresponden a las rocas que infrayacen a las areniscas y que son graníticas al $\mathrm{N}$ de playa Tanumé $\left(34^{\circ} 13^{\prime}\right)$ y esquistos al $\mathrm{S}$. Estas rocas cristalinas del zócalo casi no afloran frente a los acantilados blandos regulares al $\mathrm{N}$ de Punta Barranco y al S de Cáhuil. En cambio están presentes en toda la línea de costa rocosa irregular entre estos dos lugares.

Con estos antecedentes, la hipótesis genética puede partir de la asunción de que toda la antigua línea de costa fue de acantilados blandos y sensiblemente regular. Esta asunción se basa en el modelo evolutivo de CLAP (1913, en JOHNSON, 1919), según el cual, el retroceso continuo del acantilado blando permite la exhumación de rocas duras subyacentes que pueden corresponder a una antigua línea de costa irregular. Se contrapone asi una línea de costa regular con una irregular, razón por la que CLAPP usó la expresión "contraposed shorelines" (líneas litorales contrapuestas). Los factores estructurales generados por este proceso influyen en las características morfométricas de la línea litoral, las cuales a su vez condicionan en cierta medida el grado de estabilidad diferencial de los distintos compartimentos de las playas, o sea, la condición de estado y la condición de ataque.

\subsubsection{Definición morfológica y descripción}

Para describir y definir morfológicamente la estructura se trabajó sobre la base de los siguientes conceptos: ángulo de desalineamiento ("offset") entre la recta que pasa por dos puntas sucesivas y la que pasa por la parte más rectilínea de la playa en forma de zeta griega que media entre ellas (DAvies, 1977); asimetria o razón entre el semieje norte y el semieje sur, medidos a partir de la linea de fondo de una bahia indicando los valores bajo la mayor curvatura hacia el S (KıNG, 1977); orientación o dirección de la línea litoral en relación con la línea N S, expresada en términos de azimut; indices de curvatura o relación entre el ángulo subtendido por la playa y el valor de un radián, el cual es 1 si el ángulo subtendido es igual a 1 radián (Araya-Vergara, 1967). Estas variables fueron tratadas como un sistema de correlaciones expresado en matrices, tanto para los valores naturales de correlación como para el grado de aleatoriedad, refuerzo y réplica entre elementos de sistema. Ello dio las bases para distinguir los subsistemas espaciales.

En cada una de las playas principales se comparó perfiles transversales para varios puntos con el fin de plantear soluciones al problema de la distribución diferencial de la energía y de la materia y sus efectos en el estado de las playas.

El comportamiento de la energia del oleaje frente a ellas, fue establecido en términos de la incidencia y refracción del oleaje oceánico ("swell"), trabajando con la noción de ambiente de oleaje.

\subsubsection{Técnicas}

El bosquejo geomorfológico de la Fig. 1 está cartográficamente basado en las fotografías aéreas de marca Hycon a escala aproximada 1:70.000 y tomadas en 1955. Los topónimos y las precisiones cartográficas (coordenadas) provienen de la carta 1: 50.000 del Instituto Geográfico Militar. La información de terreno fue recogida en 1982, recorriendo exhaustivamente la linea litoral. Los puntos de observación fueron seleccionados en las fotos aéreas y traspasados a la carta topográfica para su revisión en el terreno con una ficha de observación sobre los agentes oceanográficos, el tipo de linea litoral, las formas comprometidas, la litología, la condición de ataque y el perfil de las zonas inter $y$ supramareal. Estos perfiles fueron dibujados en terreno en forma expeditiva midiendo sólo las referencias fundamentales, como microacantilados, largo de cada parte y altura de bermas. Su tipificación permite tratarlos como modelos icónicos.

En cuanto a la morfometría, el ángulo subtendido para el índice de curvatura fue medido 
excluyendo la mayor curvatura sur de las playas en zeta, porque ésto falseaba los datos al ser comparados con los de playas sensiblemente rectas pero con una gran curvatura irrelevante en su extremo, la cual también se ignoró. Tanto Araya-Vergara (1967) como Christofoletti y Pires Neto (1975 y 1980) lograron una correlación lineal perfecta y positiva entre este índice y el ángulo subtendido por la playa. Por consiguiente, midiendo este último con un buscador de radios de curvatura, el indice fue calculado mediante una recta de regresión. Para la asimetria, los semiejes fueron medidos desde la línea de playa y no desde la de costa como propone King (1977), porque ésta tiene menos significado dinámico que aquélla. El azimut fue medido centrando en el extremo sur de cada accidente litoral. Sólo para éste se usó la carta al 50.000 . Todas las otras medidas fueron hechas en la fotografía aéreas al 70 y al 30.000 .

La cinemática de los trenes de olas fue establecida en aerofotos de los veranos de 1955 y 1963 Y del otoño de 1978, midiendo la dirección de las crestas de las olas significativas.

\section{MORFOESTRUCTURA}

El sector entre Punta Barranco y Cáhuil puede ser llamado, para mayor comodidad, sector Topocalma-Pichilemu, ya que estos topónimos son más conocidos (Fig. 1). Como se desprende de la introducción, está bien individualizado por su comportamiento morfoestructural entre los sectores de acantilados más o menos regularizados de más al $\mathrm{N}$ y más al $\mathrm{S}$. Dos estilos se destaca aquí: estilo en desalineamiento ("offset") con bahias en zeta griega y estilo en desalineamiento con lineas de costa dentadas y playas rectas.

\subsection{ESTILO EN DESALINEAMIENTO [“OFFSET"| CON BAHIAS EN ZETA}

|FIG. II

Este estilo se halla en el sector de Topocalma (entre Punta Barranco y Punta Topocalma) y en el sector de Pichilemu (entre Pta. Cueva de la Negra, $34^{\circ} 19^{\prime}$, y Punta Lobos, $34^{\circ} 25^{\prime}$ ). El desalineamiento es producido por las estructuras transversales y oblicuas que dominan las rocas del zócalo. Bruggen (1950) describe líneas de falla ortogonales en Topocalma, unas N S y otras E W. CECIONI (1978), con más antecedentes regionales, muestra un patrón más complicado en que intervienen más fallas oblicuas. Esta realidad se puede resumir como una combinación entre lineamientos transversales y oblicuos y en parte longitudinales que debe ser la responsable de los desalineamientos que separan bloques cristalinos. Al N de Topocalma, en Puertecillo (34 $04^{\prime}$ ), el acantilado granítico abandonado es muy rectilineo y de dirección $\mathrm{N} S$ y presenta un ángulo de "offset" muy notable con la dirección de los acantilados y "bluffs" que hay al S de Punta Topocalma, lineamiento que queda "offset" a su vez con respecto a la punta de Pichilemu. La línea de costa entre Pichilemu y Caleta Los Piures $\left(34^{\circ} 25^{\prime}\right)$ tiene dirección N S, quedando "offset" con respecto al tramo entre Punta Lobos y Cáhuil. Las rocas del zócalo en el sector de Pichilemu corresponden a esquistos.

Debido a los ángulos de desalíneamiento, cada caso presenta una bahía en zeta griega, formada por una playa de arena asociada a un sistema de dunas. La parte sur de estas bahias tiene mayor curvatura que la norte. Este tipo de bahías fue descrito por primera vez por HALLIGAN (1906) en DAvies (1977), para Nueva Gales del Sur ("zeta curve"). Corresponde aproximadamente a las bahias de medio corazón de SILVESTER (1960), muchos de cuyos ejemplares pueden dar una buena aproximación a una espiral logaritmica según estima Krumbeim (1947) en Davies (1977), cuya sugerencia fue confirmada por YAsso (1965) en una flecha-barra de Nueva Yersey. King (1972) trata la bahía de medio corazón y la espiral logarítmica en forma separada, pero estima que son formas similares.

Las bahias de Puertecillo, Topocalma, Pichilemu y Caleta Los Piures pueden clasificarse como curvas en zeta. Las tres primeras están asociadas a sistemas dunarios importantes, con sendas estructuras diferentes, si se tiene en cuenta los grupos de dunas contenidos en cada una $y$ su distribución relativa.

\subsection{ESTILO EN DESALINEAMIENTO CON LINEAS DE COSTA DENTADAS Y PLAYAS RECTAS}

Entre Punta Tópocalma y Punta Cueva de La Negra la línea litoral cambia su dirección sensiblemente a NNW SSE (Fig. 1). Las rocas del zócalo afloran continuamente: rocas graníticas al $\mathrm{N}$ de 
Tanumé y esquistos al S. Pero la dirección de las fracturas presentạ un patrón similar en ambas unidades litológicas, en un sistema principal relativamente ortogonal NE SW y NW SE. El croquis geológico de CECIONI (1980) algo muestra sobre esta realidad, pero el estudio del drenaje y de los "offsets" litorales en la foto área es más decisivo. Como el cambio litológico producido en Tanumé no es acompañado por cambios fundamentales en la morfoestructura, se concluye en que es la estructura de las fracturas la responsable de los desalineamientos. Si se compara las playas Las Quiscas $\left(34^{\circ} 11^{\prime}\right)$. Tanumé, y Panilonco $\left(34^{\circ} 17^{\prime}\right)$ se ve un notorio desalineamiento, pero en paralelismo o con muy bajo ángulo de "offset". Las playas son fundamentalmente rectas o con muy alto radio de curvatura (difícilmente medible), arenosas y asociadas a fenómenos dunarios poco importantes.

Sólo la playa de Cáhuil se asocia a un campo de dunas importante, con estructura compleja (mixta) que contrasta con las asociadas a las bahías en zeta.

Entre las playas, la línea litoral rocosa es dentada ("crenulate" , según la denominación que KING, 1972, emplea para una parte de Devon y Cornuales). Recorriendo en terreno esta linea de costa dentada, se ve que su causa es la dirección general de diaclasas que tiende a coincidir con la de las principales fracturas ENE WSW, dando una estructura local transversal. Esta causa del carácter dentado es más notable en los esquistos al $\mathrm{S}$ de Tanumé, porque las diaclasas son comúnmente independientes de la dirección de las superficies de bandeamiento de ellos, no siendo por lo tanto la estructura esquistosa la determinante geomorfológica, sino solamente en las microformas. Es común que entre las puntas rocosas haya playas de bolsillo de cantos rodados.

\subsection{EFECTO DE LA ESTRUCTURA DISCORDANTE}

La realidad estratigráfica mostrada en la hipótesis y en la Fig. 2 es perfectamente clara en el sector de linea de costa dentada y siempre en acantilados abandonados. Entre las playas de Tanumé y Panilonco ellos presentan areniscas que es posible reconocer como lo que DARWIN (1906) llamó "formación de areniscas de Navidad" en 1846 y que actualmente CECIONI (1978) propone Ilamar Grupo Navidad. Estos sedimentos tienen un aspecto similar al de los acantilados blandos, regulares y en parte activos de la costa de Navidad y Matanzas al Norte de Topocalma y

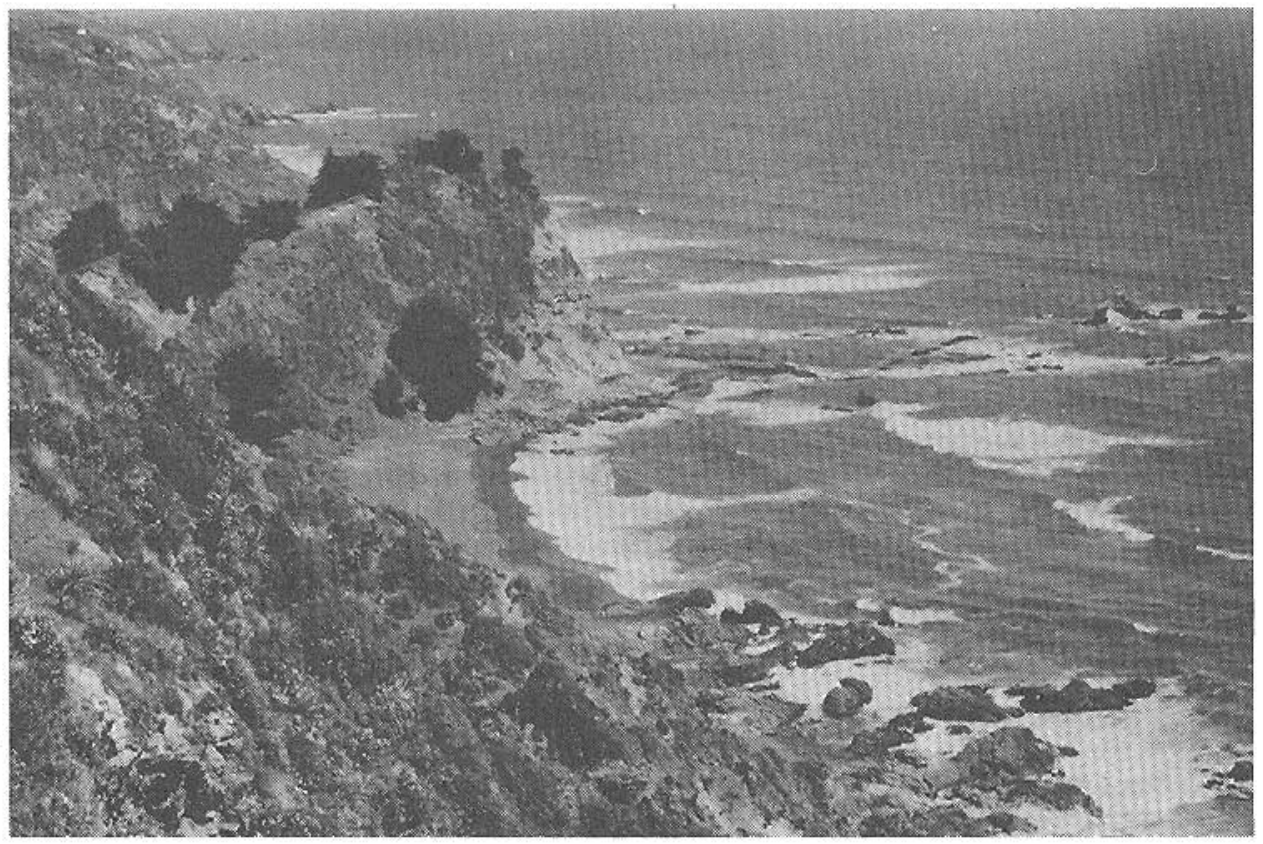

Fig. 2. Línea de costa entre Tanumé y Panilonco. Efecto de contraposición. Arriba, en acantilado, "formación de areniscas de Navidad" de DARWIN. Abajo, línea de costa dentada en esquistos del zócalo (foto Araya-Vergara). 


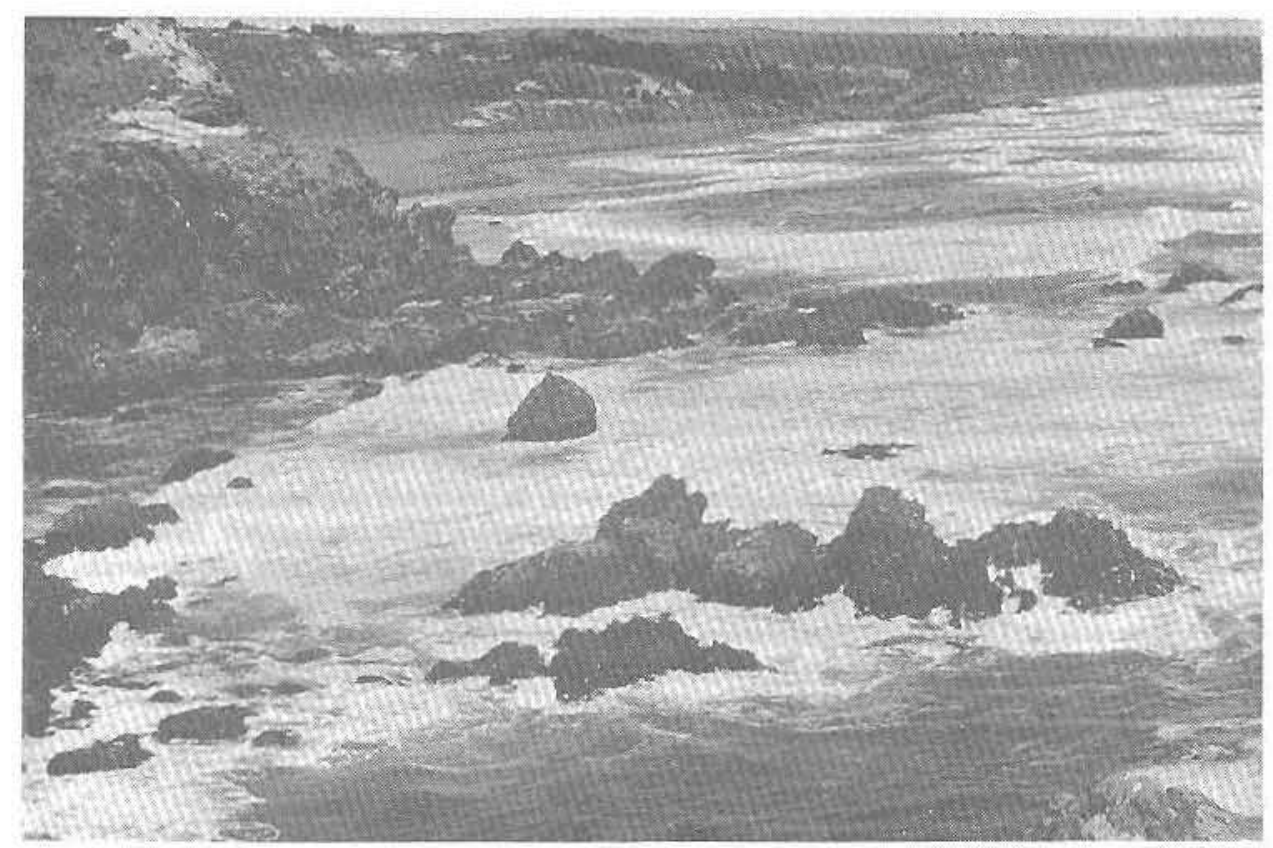

Fig. 3. Linea de costa entre Tanumé y Panilonco. Efecto de la contraposición. Puntas esquistosas y areniscas suprayaciéndolas con algunos pliegues de fondo. La concordancia de niveles escalonados en los promontorios es testigo de la existencia de antiguas plataformas de abrasión disectadas, que realzan el efecto de estructura discordante y epigénesis (foto Araya-Vergara).

Puertecillo. Los infra yacen los esquistos a media altura en el acantilado, mostrando una notable discordancia. Las puntas rocosas presentan esquistos y sólo las más altas - excepcionalmenterestos de paquetes de areniscas (Fig. 3). Tanto ellas como las entrantes están escalonadas en antiguos niveles de abrasión (Fig. 2), ya que son independientes de la estructura de los esquistos. Esto significa que el proceso de retroceso del acantilado blando, hasta más atrảs de las puntas y de los fondos de las entrantes, se realizó necesariamente sobre la base de transgresiones hasta niveles del mar más altos que el actual. Estos niveles deben poder correlacionarse con los que COOKE, PASKOFF Y WEISCHET (en FUENZALIDA et al., 1965) reconocen al $N$ y al S de esta región y que van desde 1 a $2 \mathrm{~m}$ hasta 8 a 10 sobre el nivel actual. El hecho de que las principales líneas del drenaje tiendan a coincidir con la dirección de las fracturas que condicionan la configuración dentada, puede responder a la proposición de DE MARTONNE (1926) según de que la evolución en estos casos tiene que ver con epigénesis, pues - si hay capas transgresivas correspondientes a las areniscasla red de drenaje organizada posteriormente en ellas persiste más o menos cuando la erosión ataca las rocas del zócalo en los estados regresivos. Subsecuentemente, es comprensible que el retroceso del acantilado blando, a medida que asciende el nivel del mar, haga aflorar nuevamente la roca dura que se comporta de acuerdo con su estructura. Los hechos expuestos permiten aplicar a la costa en cuestión el modelo de CLAPp citado en la introducción (en JOHNSON, 1919), agregando además el esquema epigénico propuesto por DE MARTONNE. Debido a la estructura fractural de los esquistos, el proceso dio como resultado una irregularización de la línea de costa, en oposición a la regularidad que presentan los acantilados blandos actualmente activos más al Norte y más al Sur del sector en estudio.

\subsection{DIFERENCLAS ESTRUCTURALES ENTRE LOS CAMPOS DE DUNAS}

Los campos asociados a las bahías en zeta presentan una distribución clara de los grupos de dunas. Llama la atención el predominio de dunas primarias en Topocalma y en parte en PuerteciIlo, representadas por dunas longitudinales y barjanes. Pichilemu, en cambio, tiene mayor proporción de dunas secundarias, representada por dunas parabólicas que acompañan a las transversales. Pese a las diferencias, la estructura en cada caso es fácilmente definible. En cambio, 
en la playa sensiblemente recta de Cáhuil, la aparentemente caótica mezcla de parabólicas, barjanes y transversales, hace la estructura difícilmente definible (ver mapas Fig. 1).

\section{ANALISIS MORFOMETRICO}

De acuerdo con la hipótesis, los datos brutos sobre las bahias y playas son presentados en dos cuadros (Cuadros 1 y 2), siguiendo los estilos distinguidos al tratar a morfoestructura. Si se revisan los diagramas de dispersión resultantes de cotejar los diversos pares de variables (Figs. 4 a 9), se encuentra que en la mayoría de ellos se distingue un par de poblaciones correspondientes realmente a los dos estilos morfoestructurales. Ellas son discernibles con facilidad en las relacio-

\section{Cuadro 1: Propiedades morfométricas}

Estllo en desalineamiento con bahías en zeta

Orientación general (azimut): $32^{\circ}-33^{\circ}$

\begin{tabular}{|c|c|c|c|c|c|}
\hline Playa o bahia & $\underset{0}{\text { Latitud }}$ & $\begin{array}{l}\text { Ángulo "offset" } \\
\text { (Of) }{ }^{\circ}\end{array}$ & $\begin{array}{l}\text { Asimetría } \\
(\mathrm{A})\end{array}$ & $\begin{array}{l}\text { Azimut } \\
(\mathrm{Az})^{\circ}\end{array}$ & $\begin{array}{l}\text { Indice de } \\
\text { curvatura } \\
\text { (C) }\end{array}$ \\
\hline Puertecillo & 3404 & 28 & 0.84 & 27 & 0.77 \\
\hline Cta. Sto. Dgo. & 3405 & 42 & 0.38 & 29 & 2.01 \\
\hline Topocalma & 3407 & 30 & 0.39 & 24 & 0.58 \\
\hline Pichilemu & 3422 & 25 & 0.89 & 22 & 0.89 \\
\hline Infiernillo $\mathbf{N}$ & 3423 & 21 & 0.33 & 21 & 0.28 \\
\hline Infiernillo S & 3423 & 15 & 0.62 & 24 & 0.41 \\
\hline $\begin{array}{l}\text { Pl. Hermosa-Cta. } \\
\text { Los Piures }\end{array}$ & 3425 & 31 & 0.33 & 17 & 0.28 \\
\hline Promedio & & 27 & 0.54 & 23 & 0.75 \\
\hline
\end{tabular}

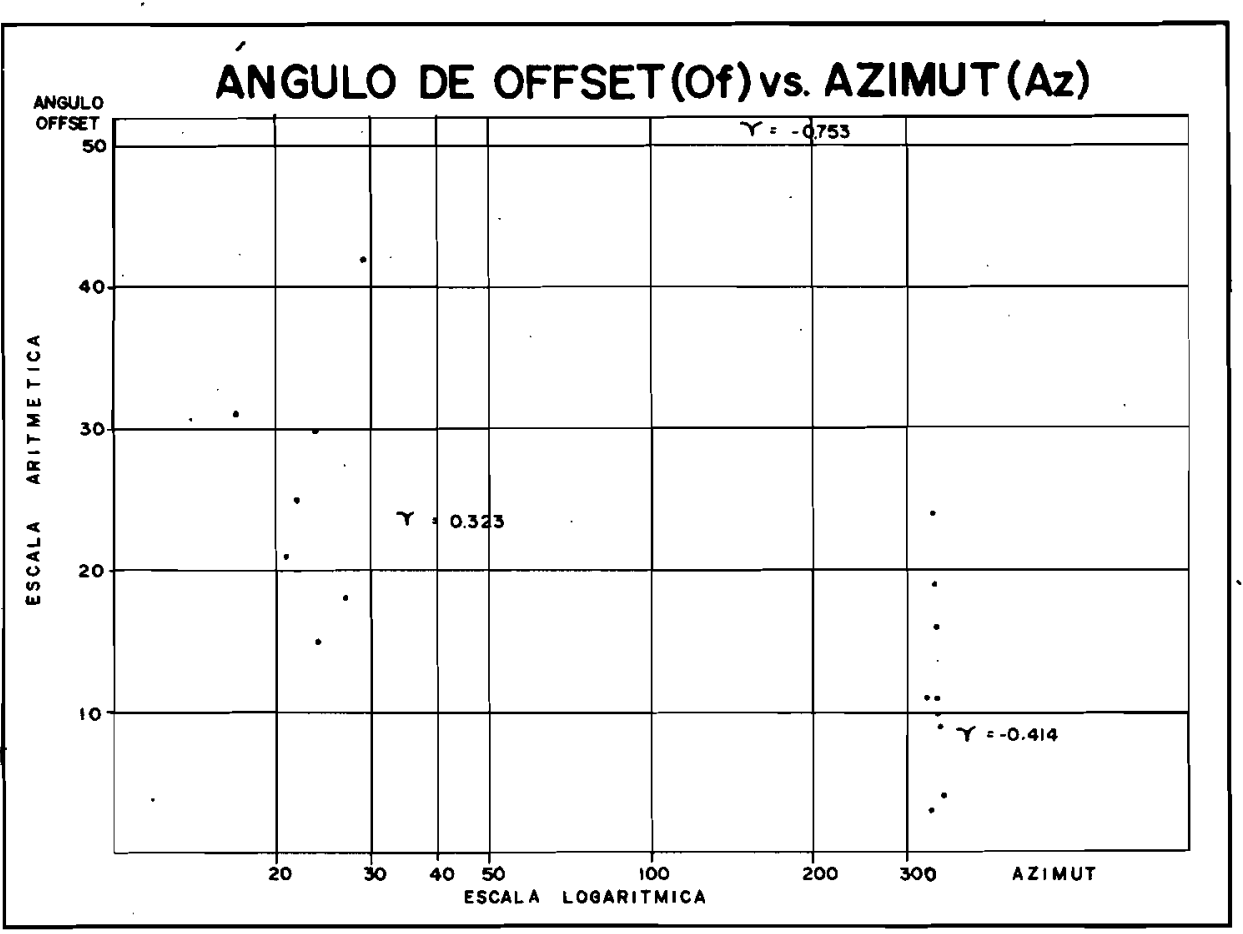

Fig. 4 
nes siguientes: ángulo de "offset" vs. azimut, índice de curvatura vs. azimut y asimetria vs. azimut. Para la relación asimetría vs. ángulo de "offset" no es posible separar poblaciones. La separación sólo por dispersión de los puntos no puede hacerse con facilidad para las relaciones indice de curvatura vs. asimetría e índice de curvatura vs. ángulo de "offset", pero ella es encontrada fácilmente si se encierra cada población, puesto que los límites no se interpenetran, como sucede en la relación asimetría vs. ángulo de offset.

A pesar de que dos poblaciones son discernibles en los diagramas, los análisis de correlación y de matrices permitirán entender mejor el papel de cada una de las variables en la caracterización de los estilos.

Cuadro 2: Propiedades morfométricas

Estllo en desalineamiento con líneas dentadas y playas sensiblemente rectas Orientación general (az): $334-351^{\circ}$

\begin{tabular}{lccccc}
\hline Playa o bahia & Latitud & Of $^{\circ}$ & A & A $\mathbf{Z}^{\circ}$ & $\mathrm{C}$ \\
\hline Topocalma S & 3408 & 10 & 9.60 & 341 & 0.07 \\
Cta. Quincheumo & 3410 & 11 & 0.89 & 340 & 0.05 \\
Playa Las Quiscas & 3411 & 16 & 0.32 & 340 & 0.07 \\
Playa Tanumé & 3413 & 3 & 0.78 & 335 & 0.07 \\
Tanumé S & 3414 & 4 & 0.38 & 352 & 0.16 \\
Q. Deslinde & 3414 & 24 & 0.86 & 339 & 0.16 \\
Centinela N & 3415 & 19 & 1.00 & 333 & 0.10 \\
Centinela N & 3415 & 11 & 1.00 & 338 & 0.10 \\
Playa Panilonco & 3417 & 9 & 0.47 & 347 & 0.14 \\
Playa Cáhuil & 3427 & 9 & 0.95 & 345 & 0.21 \\
Promedio & & 12 & 1.62 & 341 & 0.11 \\
\hline
\end{tabular}

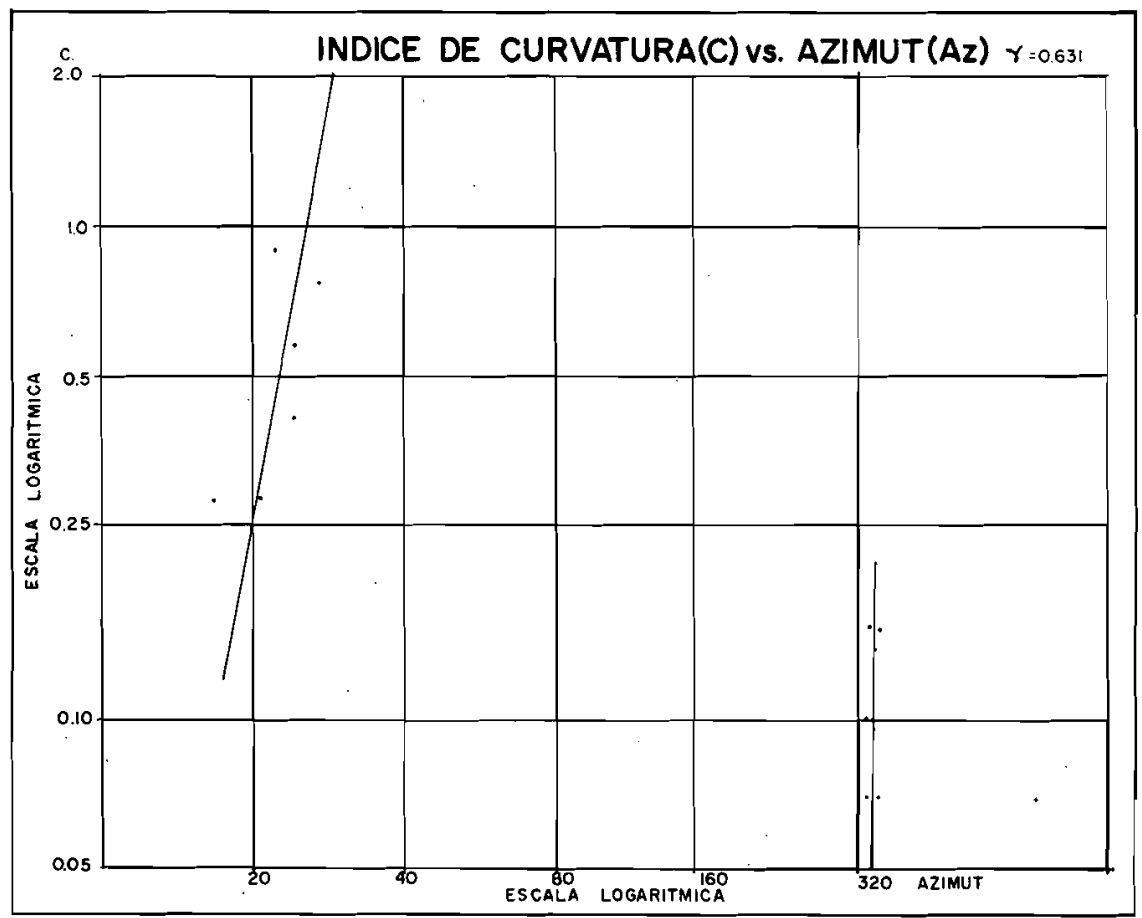

Fig. 5 


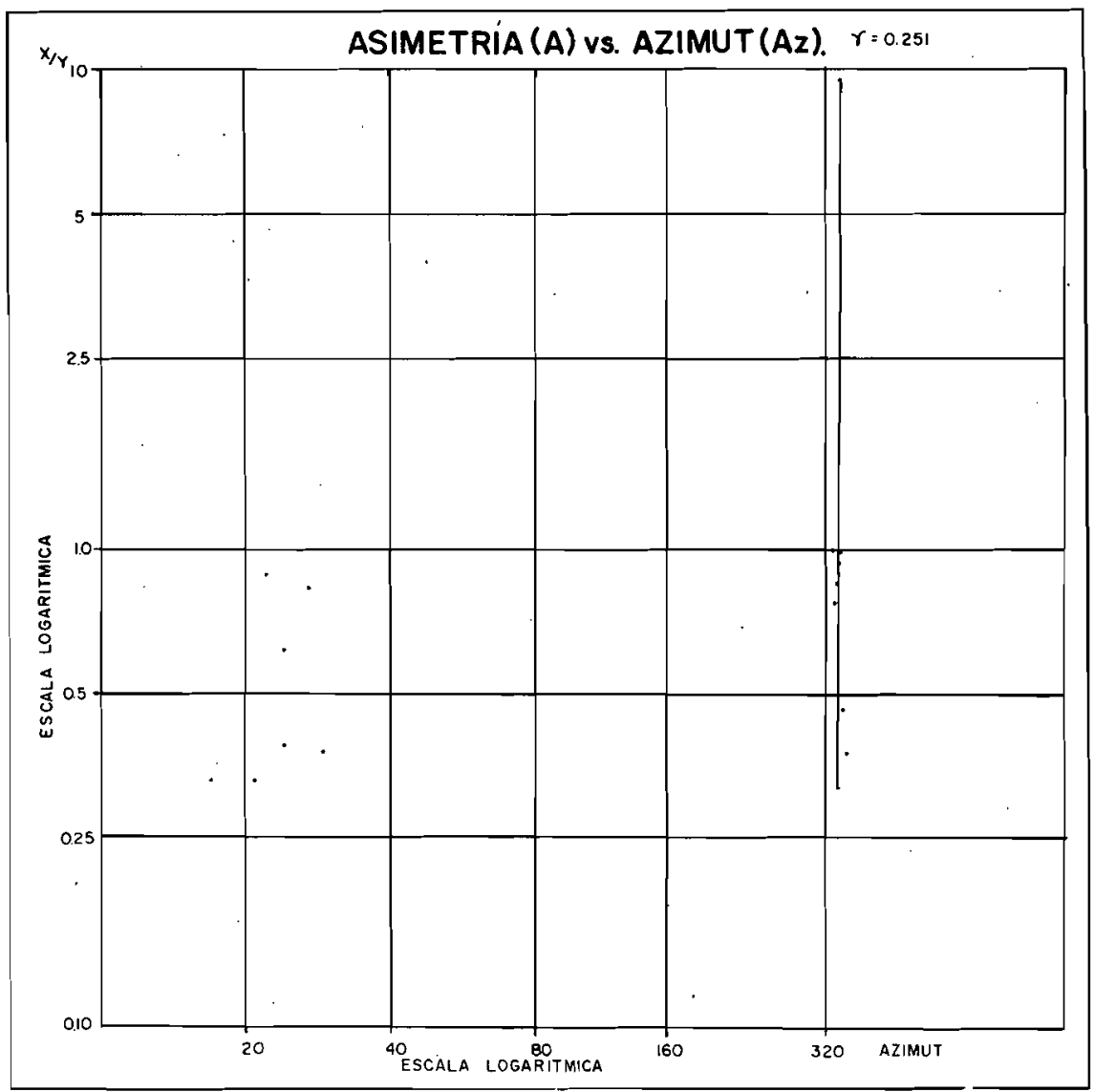

Fig. 6

\subsection{ANALISIS DE CORRELACION}

Revisando los promedios de cada una de las variables consideradas para el análisis sin distinción de poblaciones, se constata que los coeficientes de variación respectiva son demasiado grandes (Cuadro 3), si se toma en consideración la simple comparación con el valor de los primeros. A juzgar por la observación de los gráficos de dispersión, es fácil sospechar que los coeficientes altos están influidos por la existencia de más de una población y que, por lo tanto, el uso de promedios generales no tiene sentido. Por esta razón vale más la pena apreciar los promedios puestos en los Cuadros 1 y 2 que reflejan una notoria separación entre poblaciones, para ambos casos de desalineamiento.

Cuadro 3: Datos generales

\begin{tabular}{lccc}
\hline & $\bar{x}$ & $\sigma$ & $V$ \\
\hline${\text { Azimut }(A z)^{\circ}}^{\circ}$ & 210 & 156 & 74 \\
"Offset" (Of) & 18 & 10.4 & 57 \\
Indice curvatura (C) $_{\text {Asimetria (A) }}$ & 0.37 & 0.48 & 129 \\
\hline
\end{tabular}

Esta separación hipotéticamente debe influir en la correlación entre componentes y, por lo tanto, hay que detener la atención en el sistema de correlaciones. De la matriz de correlación, del 


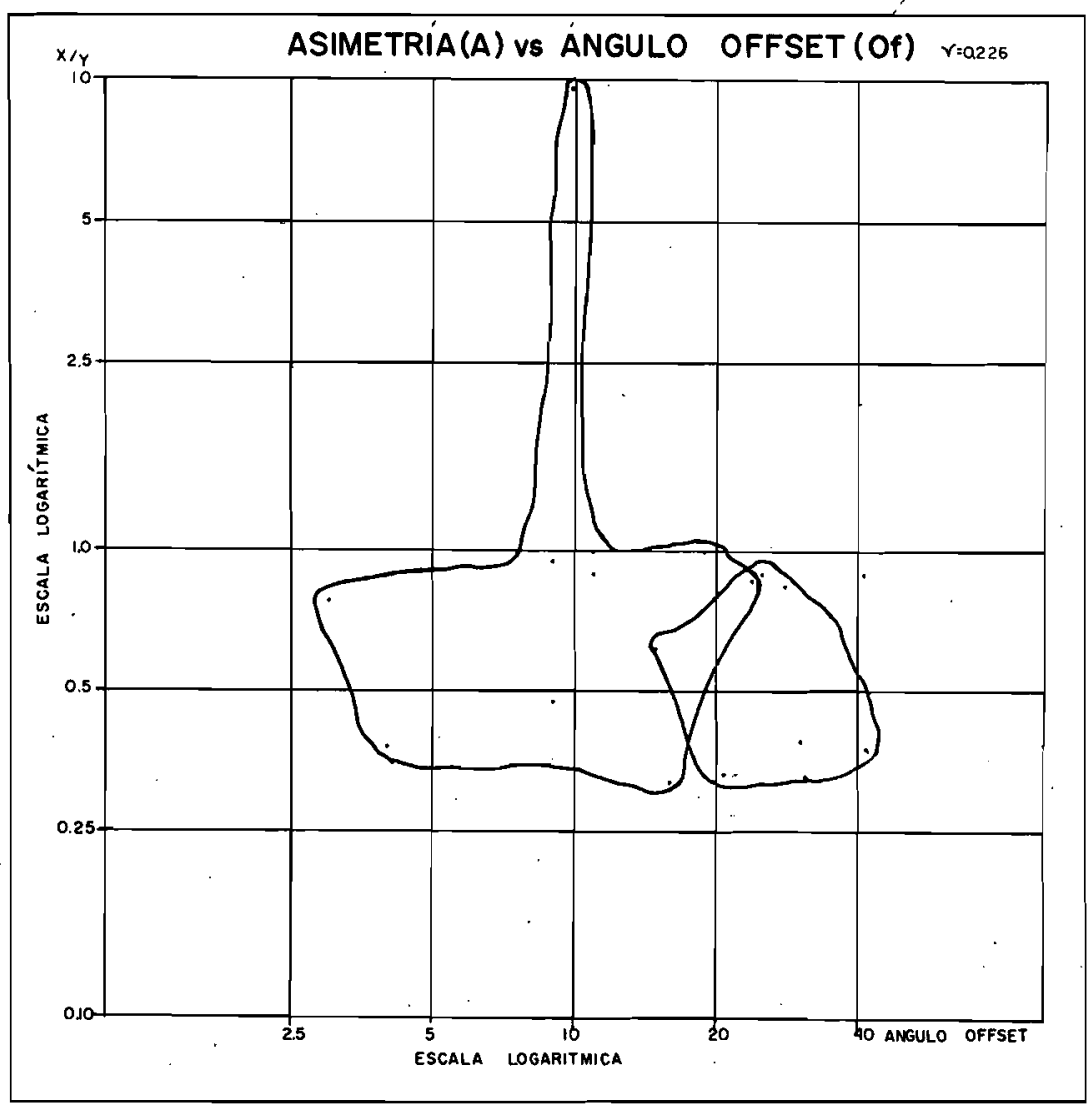

Fig. 7

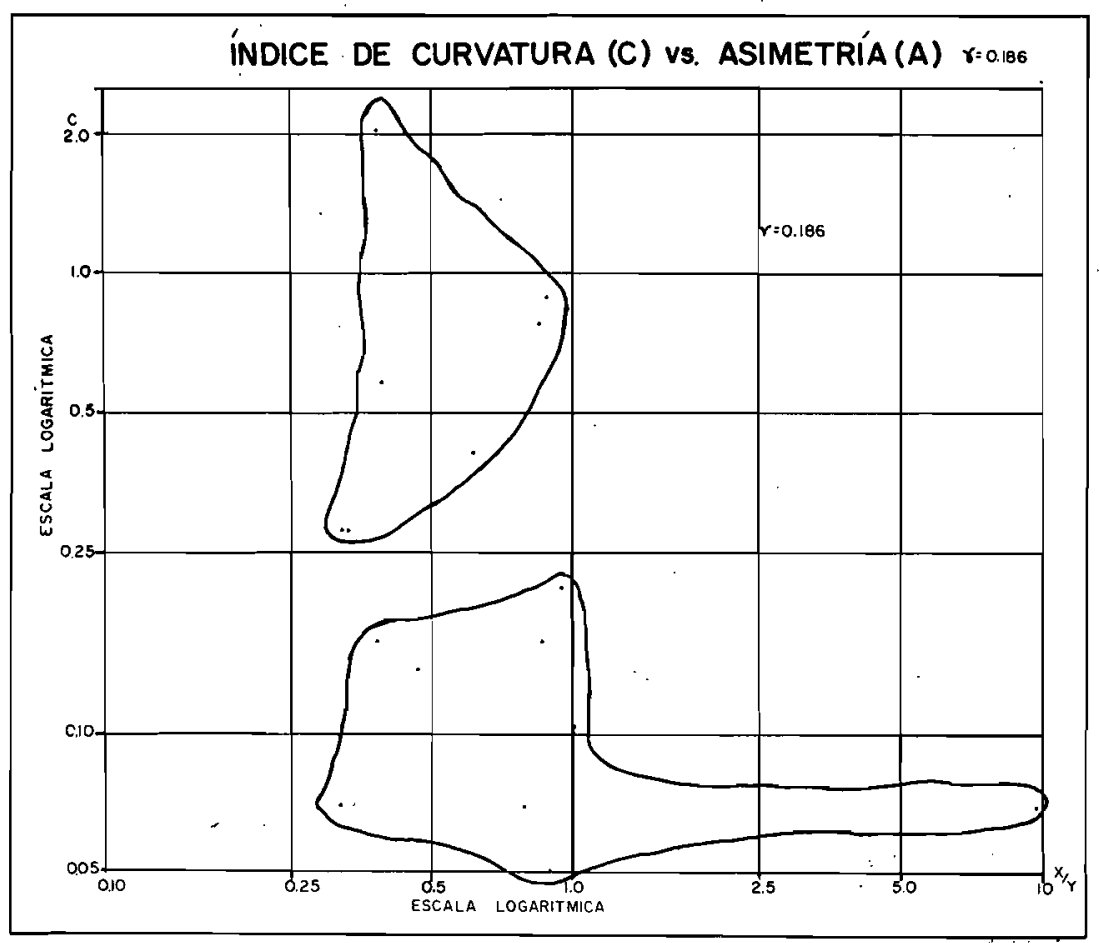

Fig. 8 


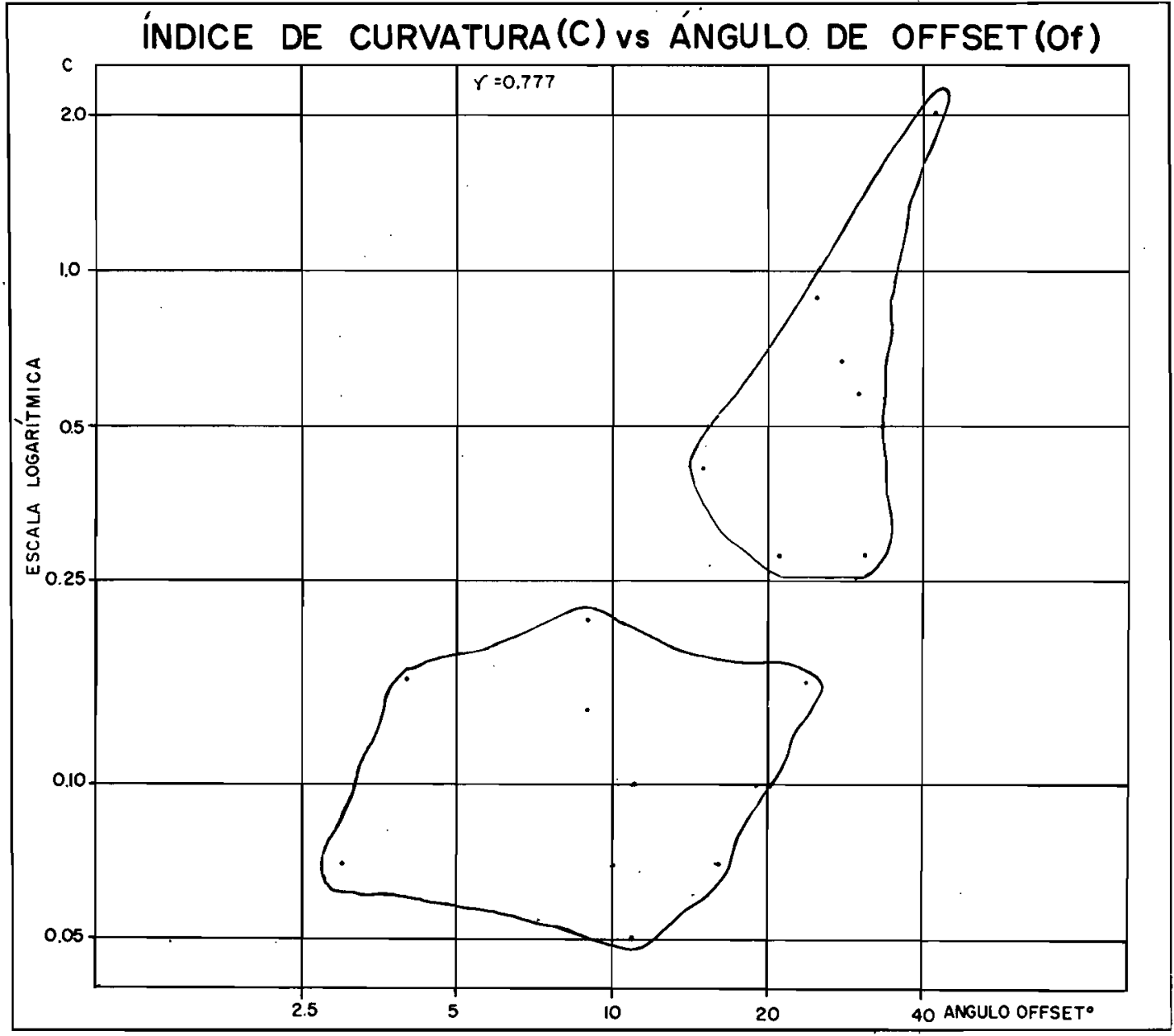

Fig. 9

Cuadro 4 se desprende que entre algunos elementos hay una correlación media a fuerte, cuya tendencia tiene que estar de acuerdo con las características de cada uno de los estilos representados por las poblaciones. Así, con el objeto de reconocer en torno a qué asociaciones debe estar basada la distinción de estilos, la correlación está hecha en el total y no por poblaciones.

\section{Cuadro 4: Matriz de correlación general (r)}

\begin{tabular}{lrccc}
\hline & \multicolumn{1}{c}{$\mathrm{Az}$} & Of & \multicolumn{1}{c}{$\mathrm{C}$} & $\mathrm{A}$ \\
\hline $\mathrm{Az}$ & - & -0.753 & 0.251 & \\
Of & -0.753 & - & -0.226 & \\
$\mathrm{C}$ & -0.639 & 0.777 & -0.186 & \\
$\mathrm{~A}$ & 0.251 & -0.186 & - & \\
\hline
\end{tabular}

Se ve que el azimut, el ángulo de "offset" y el índice de curvatura son los componentes decisivos que sirven para establecer atributos de subsistema. Comparando la matriz con las Figs. 4,5 y 9 se ve que la alta relación negativa entre azimut y ángulo de "offset", la regularmente alta relación negativa entre azimut e índice de curvatura y la fuerte relación positiva entre ángulo de "offset" $e$ índice de curvatura indican que es la asociación de estos tres factores la que permite identificar cada estilo por separado. Las otras correlaciones son débiles y desechables como 
elementos de distinción. Esta realidad queda establecida claramente trabajando con la matriz del Cuadro 5 que permite expresar la aditividad ("addivity") mediante la suma de los cuadrados de las correlaciones posibles de cada variable. Así, la simetría exhibe aditividad (aleatoriedad), ya que la suma de $r^{2}$ es baja (mucho menor que la unidad), frente a la no aleatoriedad del azimut, el ángulo de "offeset" y el indice de curvatura. Por lo tanto, siendo este sistema de correlación no aditivo en lo fundamental, mostrando interacción entre las tres principales variables, cada una de éstas crece en efectividad como resultado de su operación en conjunción con las otras: hay efecto de refuerzo. En cambio, las correlaciones con la asimetría indican sólo un efecto de réplica en el sistema.

Si se ordena jerárquicamente las sumas de $r^{2}$, se ve que la variable en torno a la cual hay más refuerzo es el ángulo de "offset"; después están el índice de curvatura y el azimut. Este orden, por lo tanto, servirá de base para separar los estilos y realizar su descripción morfométrica.

Cuadro 5: Matriz de correlaclón general: determinación $\left(\mathbf{r}^{2}\right)$

\begin{tabular}{lccccc}
\hline & $\mathrm{Az}^{2}$ & $\mathrm{Of}^{2}$ & $\mathrm{C}^{2}$ & $\mathrm{~A}^{2}$ & $\Sigma \mathrm{r}^{2}$ \\
\hline $\mathrm{Az}^{2}$ & - & 0.567 & 0.408 & 0.063 & 1.038 \\
$\mathrm{Of}^{2}$ & 0.567 & - & 0.604 & 0.051 & 1.222 \\
$\mathrm{C}^{2}$ & 0.408 & 0.604 & - & 0.035 & 1.047 \\
$\mathrm{~A}^{2}$ & 0.063 & 0.051 & 0.035 & - & 0.149 \\
\hline
\end{tabular}

Los coeficientes de determinación individuales demuestran que las varianzas de cada uno de los tres componentes principales son explicadas entre un 40 y un $60 \%$ aproximadamente por la varianza de los otros dos. Este último porcentaje corresponde a la determinación entre ángulo de "offset" e indice de curvatura.

Revisando la matriz de significación del Cuadro 6 y siendo el valor crítico de 1.75 para 15 grados de libertad al nivel de significación de $0.1 \%$, resulta que se puede afirmar, para los tres componentes principales, la hipótesis alternativa de que su correlación es significativa. En cambio, para las correlaciones con la asimetria, se corrobora la hipótesis nula acerca de que ellas provienen del azar.

Cuadro 6: Matriz de signifleación: valores de $t$ (Valor crítico: 1.75 para 15 grados de libertad)

\begin{tabular}{lcccc}
\hline & Az & Of & C & A \\
\hline Az & - & 4.43 & 3.21 & 1.00 \\
Of & 4.43 & - & 4.78 & 0.89 \\
C & 3.25 & 4.78 & - & 0.73 \\
A & 1.00 & 0.89 & 0.73 & - \\
\hline
\end{tabular}

Es evidente, entonces, que la descripción separada de los estilos debe hacerse sobre la base de los tres componentes principales.

\subsection{DESCRIPCION DE LOS ESTILOS}

\subsubsection{Estilo en desalineamiento con bahías en zeta}

La línea de costa tiene aqui una orientación general de 32 a $33^{\circ}$. Los desalineamientos van de 21 a $42^{\circ}$, promediando en 27 . Los índices de curvatura se presentan medianos a bajos (entre 0.28 y 2.01, promediando en 0.75 ), considerando que sólo un caso pasa de 1 (arco subtendido igual a 1 radián). Esto se debe a que no se consideró en las medidas la curvatura mayor del extremo sur de las bahías en zeta lo que aumenta considerablemente las curvaturas, pero no representa la generalidad. Considerando la dirección parcial de los sectores principales de playas, los azimutes aparecen entre 17 y $29^{\circ}$, promediando en 33. La asimetría es siempre inferior a 1 y, a veces, muy 
inferior, lo que significa que siempre la mayor curvatura está hacia el Sur, siendo el promedio 0.54 .

Las Figs. 4, 5 y 9 indican correlaciones positivas entre los tres componentes principales, pero no siempre altas, como es el caso de ángulo de "Offset" vs. azimut. El índice de curvatura, en cambio, se correlaciona bien con el azimut y medianamente bien con el ángulo de "offset".

\subsubsection{Estllo en desalineamiento con líneas litorales dentadas y playas senslblemente rectas}

La orientación general de esta línea litoral es de 334 a $351^{\circ}$. Los desalineamientos están entre 3 y $24^{\circ}$, promediando en 12 . Los índices de curvatura son muy bajos y van de 0.05 a 0.21 , promediando en 0.11. Considerando la dirección parcial de los sectores principales de playas, los azimutes aparecen entre 335 y $345^{\circ}$, promediando en 341 . La asimetría muestra un comportamiento anárquico, promediando en 1.62, pero con más del $90 \%$ de la varianza no explicada, como se desprende en el Cuadro 5.

Las Figs. 4, 5 y 9 indican débiles correlaciones dentro del estilo. La relación entre índice de curvatura y ángulo de "offset" no muestra correlación lineal. La correlación entre estas variables y el azimut casi no puede tomarse en cuenta, debido a la escasa variación práctica de este último.

\section{AMBIENTE DE OLEAJE Y PERFILES DE PLAYAS}

De la determinación preliminar de las características del ambiente de oleaje para estas latitudes (Araya-Vergara, 1971), se sabe que el oleaje oceánico ("swell") más significativo es el del S.W. Se estima que para Constitución, un grado más al Sur que el sector estudiado, este componente predomina en verano y en estaciones medias con relación a las olas forzadas locales (mar local) y en verano $y$ en invierno para el oleaje oceánico. En estación transicional el oleaje oceánico proviene más bien del Oeste. En invierno, el mar local más importante es el del Norte (TUDOR ENG. Co., 1965). Para San Antonio, 30' más al Norte que el sector estudiado, se mantiene la predominancia SW. (Ministerio de Obras Públicas y Dirección de Obras Portuarias, 1953-54) especialmente en verano $y$ con tendencia $w$ en invierno, presumiblemente para el oleaje oceánico. $\mathrm{El}$ azimut de las ortogonales a las crestas del oleaje oceánicos del S.E. es comúnmente $25^{\circ}$ (DIRección de Obras Portuarias y Tudor Eng. Co. 1968).

Cuadro 8: Relación entre la dirección del oleaje de aguas profundas y la direcelón de la línea litoral

\begin{tabular}{lllc}
\hline $\begin{array}{l}\text { Dirección desde la cual } \\
\text { las olas se aproximan (az) }\end{array}$ & \multicolumn{2}{l}{ Ángulo teórico de incidencia con la línea litoral $\left(^{\circ}\right)$} \\
\hline & & En litoral con Az: $23^{\circ}$ & En litoral con Az: $341^{\circ}$ \\
\cline { 2 - 3 } N & $000^{\circ}$ & 23 & 0 \\
NW & $315^{\circ}$ & 68 & 26 \\
W & $270^{\circ}$ & 67 (desde el S) & 71 (desde el N) \\
SW & $225^{\circ}$ & 22 & 64 \\
\hline
\end{tabular}

A pesar de que las propiedades de la ola y la profundidad del fondo son factores esenciales de la refracción de su dirección original de aguas profundas, también lo es la relación entre la dirección original y la de la línea litoral. La tendencia a refracción es inversamente proporcional al ángulo de incidencia de las olas, suponiendo condiciones homogéneas del fondo. Del Cuadro 8 se deduce una enorme diferencia de incidencia teórica de los trenes de olas de diferentes direcciones si se toma separadamente a los estilos morfoestructurales estudiados, por lo que las condiciones de refracción deben variar bastante. Esto se comprueba estudiando fotos aéreas de distintas fechas: verano de 1955, verano de 1963 y otoño de 1978. La dirección del oleaje oceánico (ola significativa) es en todas $\mathbf{S} W$.

Del Cuadro 9 se observa que frente a las bahias en zeta hay mucho más refracción que frente a las playas sensiblemente rectas. En las Figuras 10 y 11 se ve que la refracción varía en forma directa con la curvatura y el ángulo de "offset". Esto quiere decir que las crestas de los trenes de olas oceánicas se encorvan proporcionalmente a la curvatura de las bahías y playas, lo que causa un ataque distinto para cada estilo. Pero lo más importante es destacar las consecuencias que esto 


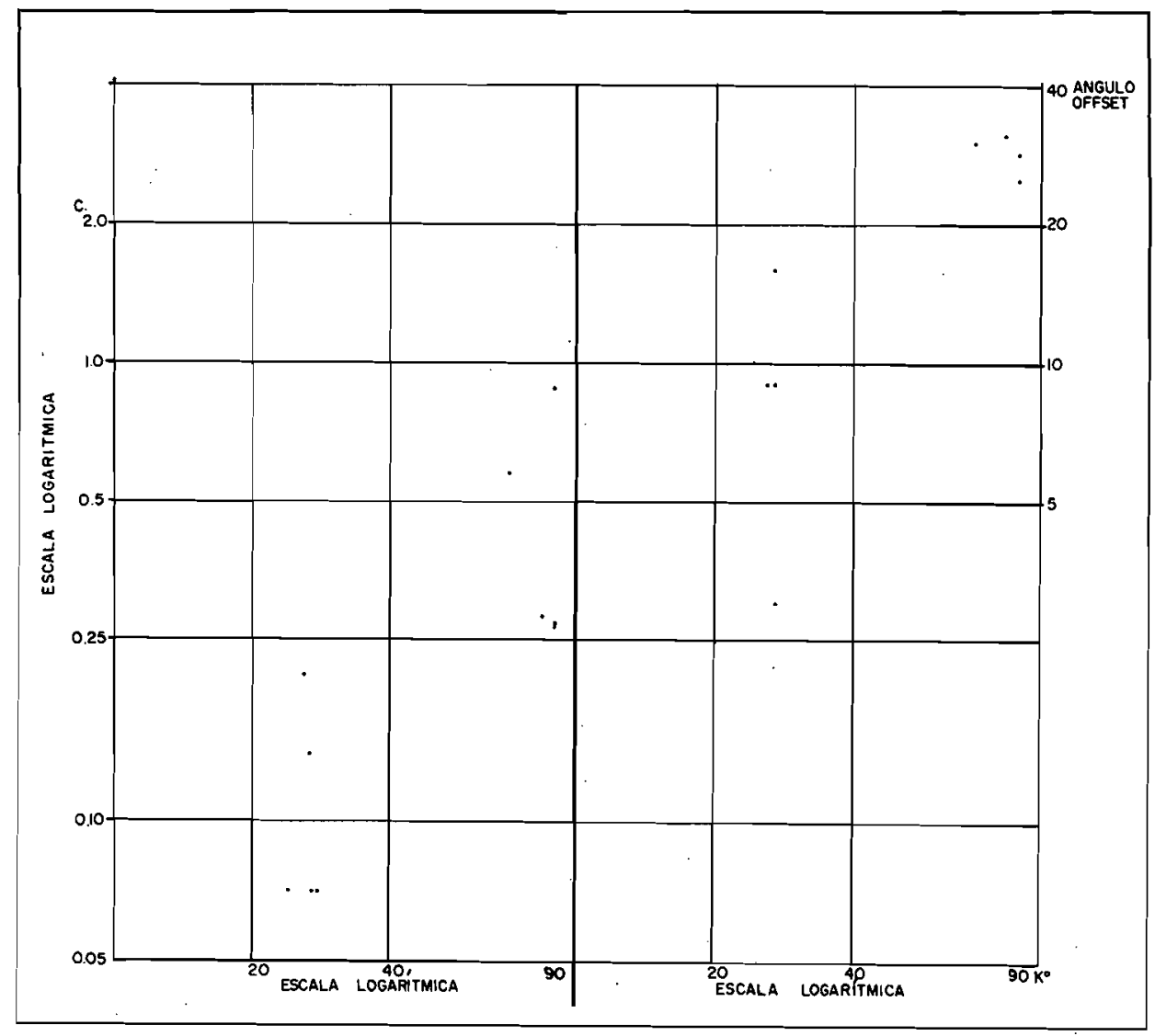

Fig. 10

Fig. 11

Cuadro 9: Refracclón del oleaje oceánico del S.W.

\begin{tabular}{lrll}
\hline \multicolumn{2}{l}{ En estilo con bahías en zeta } & $\begin{array}{l}\text { En estilo con playas } \\
\text { sensiblemente rectas }\end{array}$ \\
\hline Puertecillo & $90^{\circ}$ & Topocalma S. & $24^{\circ}$ \\
Topocalma & $72^{\circ}$ & Playa Las Quiscas & $27^{\circ}$ \\
Pichilemu & $90^{\circ}$ & Playa Tanumé & $27^{\circ}$ \\
Playa Hermosa-C. & & Playa Panilonco & $27^{\circ}$ \\
Piur. & $85^{\circ}$ & Playa Cáhuil & $26^{\circ}$ \\
\hline
\end{tabular}

trae en el ataque diferencial dentro del estilo. La observación detallada de terreno muestra que en las playas en zeta la energía del oleaje oceánico se reparte desigualmente, durante las bravezas o marejadas. La prospección de noviembre de 1982, después de un invierno particularmente tormentoso, indica que los perfiles de playa revelan mayor degradación en la parte más curva del Sur de la zeta (ver perfiles en Fig. 1). Se ve aquí el mayor truncamiento del perfil del cordón litoral, como en Cta. Tumán (Puertecillo) o en Pichilemu, o simplemente erosión directa de las dunas marginales, como en Caleta Los Piures. El perfil de anteplaya queda cóncavo en estos casos, al menos hasta fines de primavera. Detrás del micro acantilado permanece un residuo de postplaya o simplemente la duna. En los perfiles de la parte más abierta de la bahía, este fenómeno es restringido, como en Puertecillo, Pichilemu Norte y Playa Hermosa, o simplemente nulo o muy restringido, como en Topocalma, donde la tendencia convexo-cóncava de la anteplaya revela más bien una situación deposicional.

En el estilo con playas sensiblemente rectas, las anteplayas indican comúnmente un perfil convexo-cóncavo, aunque aparezcan microacantilados hacia la postplaya, altaplaya o duna marginal. Hay una mayor tendencia a conservarse la forma de un cordón litoral. Los perfiles 
cóncavos que suelen aparecer para anteplayas están delimitados por microacantilados pequeños (máx $50 \mathrm{~cm}$ ), indicando baja tendencia erosiva. Su ubicación en la playa no depende de la mayor curvatura, como en el otro estilo, sino de condiciones locales, como efecto de desembocadura o roquerios.

\section{DISCUSION}

\subsection{SOBRE LA CUESTION DE LA LINEA GENETICA}

Seguir el modelo evolutivo de CLAPP para explicar el papel de la contraposición en los desalineamientos, implica el manejo de la hipótesis ergódica. Según ella, se asume que el sector estudiado -en una fase evolutiva anterior a la actual- debe haber sido más regular por el predominio de los sedimentos blandos como los que actualmente conforman el cuerpo de los acantilados de más al $\mathrm{N}$ y más al $\mathrm{S}$. Se asume, por consiguiente, que no hay ninguna razón para que - con un nivel del mar más bajo- las formas de la línea de costa no tuvieran su cuerpo constituido por sedimentos blandos como en otros sectores muy cercanos. Pero esta asunción no permite por si sola manejar la proposición de CLApp sin tenerse en cuenta los conocimientos sobre evolución del nivel del mar, morfoestructura y estratigrafía, dados a conocer más atrás.

Si puede aceptarse la hipótesis de irregularización por el mecanismo deducido anteriormente, quiere decir que el bosquejarse las nuevas bahías, el proceso de formación de las playas correspondientes debe haber estado relacionado con el carácter epigenético de la costa. El cambio paulatino de los atributos de sistema, debe haber implicado el que la red de drenaje epigénica tuviera que ver en el abastecimiento de las nuevas playas. Usando el método experimental, Silvester (1960) produjo en un tanque, playas en forma de zeta con un oleaje incidente en $45^{\circ}$. Cuidó de no causar alimentación de arenas en el extremo de deriva arriba de las bahías. Consiguió así una forma que él llamó de "medio corazón" ("half-heart shape"). El lado de deriva abajo fue alineado más o menos perpendicularmente a la dirección de aproximación de las olas de agua profunda, siendo poco refractadas. En el lado de deriva arriba hubo refracción y difracción que curvaron la playa. Se advirtió transferencia de sedimentos de un medio corazón a otro por deriva. Esto indica que, para el caso analizado aqui, unas pocas cuencas epigenéticas deben haber bastado para asegurar el abastecimiento en cadena de las playas. De hecho hay aquí tres cuencas principales de importantes sedimentológica que tienen su exutorio en las playas de Topocalma, Pichilemu y Cáhuil. Según el Cuadro 8, el ángulo de incidencia teórico del oleaje del SW para los sectores con playas en zeta es de $22^{\circ}$, más favorable a la deriva que en el caso del experimento de SiLVESTER. Pero el mismo ángulo para las playas sensiblemente rectas es menos favorable $\left(64^{\circ}\right)$. La transferencia debe ser más factible entonces en las bahías en zeta.

\subsection{EL PROBLEMA DE LA DESIGUALDAD DE ATAOUE EN LAS BAHLAS}

Levantamientos en playas en zetas australianas (DAVIES, 1977) revelan, en las mayores curvaturas sur, bermas más bajas con arenas más finas y pendientes de playa menores que en los sectores norte más expuestos y rectilineos. Esto es interpretado como una respuesta al aumento de la energía de la ola hacia el Norte en condición de transporte largamente impedido. Las desembocaduras tienden a ser deflectadas por vaivén ("swash") y ocurren donde la construcción de bermas es más débil. La mayor parte de las bahias en zeta muestra la desembocadura en el Sur, a pesar de las condiciones generales de deriva hacia el Norte en el litoral. Las arenas son bien gradadas longitudinalmente, lo que debe esperarse en estas formas esencialmente alineadas por vaivén.

Las observaciones de KING (1977)en Chile concuerdan aproximadamente con esas generalizaciones. Según ellas, la energía del oleaje tiende a ser mayor en las secciones norte de las playas. Por otra parte, el $75 \%$ de los casos observados presenta deflexiones fluviales hacia el Sur. Las bahias muestran comúnmente su mayor profundidad hacia el extremo sur, lo que contribuye a explicar la mayor concentración de la energía hacia el Norte. Pero para esto, la base de observación fue la carta náutica y no el terreno ni las fotos aéreas.

Para tratar de armonizar estos resultados con los del presente trabajo hay que recordar lo analizado en el punto 4, sobre oleaje y perfiles de playa. Según esto, hay muestras de mayor energía erosional en la parte más curva, pero a su vez de buena energía deposicional en la parte más recta. Esto está en aparente contradicción con la teoría acumulada para las bahías en zeta y su interpretación es delicada. La experiencia de YAsso (1965) para playas de bahia de saliente ("headland bay beach") concuerda más, en cambio, con los resultados encontrados recorriendo 
los casos chilenos. Según ella, el plano cóncavo junto a la saliente es debido a erosión causada por refracción, difracción y reflexión de olas en la zona de sombra detrás de la punta. Esto explica que el radio de curvatura aumenta con la distancia a la punta rocosa, tomando la curva general la forma de una espiral logarítmica, que puede ser calzada con la curva matemática por un proceso computacional. Esto conduce a que una interpretación más exacta debe conseguirse si se atiende a la temporalidad de los procesos. Recordando que las observaciones de terreno en Chile fueron realizadas en la primavera de 1982, después de un invierno anormalmente tormentoso en el Pacífico del Sur, es natural pensar que las condiciones encontradas se deben a umbrales. Si la condición media de agitación en la parte más cóncava de una bahía indica energía relativamente baja, no ocurre lo mismo en marejadas, donde los trenes precursores tienen largos periodos, refractando más y con mayor energía, ya que las ortogonales, que tienden a converger hacia las puntas, concentran también energia en la playa cóncava inmediata. Esto no podría ser de otra manera, porque la refracción observada en las aerofotos de distintas fechas muestra que la espiral logarítmica tiende a repetirse también en la forma de las crestas de las olas antes de romper. Si las alturas aumentan, dada una mayor deformación de los precursores más largos al comenzar las marejadas, el efecto erosivo en la parte sur debe ser mayor. Análisis de regresión simple y múltiple para playas de Rhode Island mostraron a SAKaLowsKy (1975) que la altura de la ola es el más importante elemento de proceso que causa cambios en la configuración de la playa, estando el $42 \%$ de los efectos relacionados con ella. Esto debe armonizarse con la observación experimental de Silvester (1960) que indica que en el lado de deriva arriba de las bahias de medio corazón hubo refracción y difracción que curvaron la playa. Si estos procesos pueden curvar la playa quiere decir que son consubstanciales con las alturas de las olas significativas. De otra manera, no podría curvarse más un sector que otro. A esto se puede agregar una experiencia del verano de 1983, en que hubo marejada en la costa de Chile Central y del Norte, fenómeno fuera de lo común, porque las marejadas son en invierno. Cuando la marejada del mes de febrero estaba terminando, fue posible observar en continuo las playas de Cachagua y Maitencillo $\left(32^{\circ} 28^{\prime}\right)$, mucho más al Norte que el sector estudiado. La parte media de la playa de Maitencillo fue atacada dejándola húmeda por algunos dias hasta la duna marginal, pero sin señales de degradación notoria. La parte sur más curvada dejó perfil cóncavo de anteplaya y notorias condiciones de promiscuidad para los bañistas.

Por otra parte, las playas en zeta no parecen deberse básicamente al alineamiento por vaivén, como propone DAVIES (1977). La revisión de terrenos de los casos chilenos permite proponer que el proceso responsable de la configuración no es tan simple. De 17 lugares observados en las cuatro bahías en zeta más importantes estudiadas, 3 presentaron características de vaivén (la concavidad máxima de Playa Infiernillo en Pichilemu y dos en la parte norte de Playa Hermosa); 4, una combinación entre deriva y vaivén (las concavidades máximas de Pichilemu y de Cta. Los Piures y dos lugares en la parte media y norte de Playa Hermosa). Los 10 lugares restantes, esencialmente en la parte media y norte de la zeta mostraron alineamiento por deriva. El criterio de distinción fue la presencia o ausencia de "beach cusps". Vuelve a recordarse que la observación fue hecha en primavera y después de un invierno de repetidas marejadas y en estados de mar correspondientes a mar rizada y marejadilla. Para la interpretación de esto hay que destacar la importancia de la orientación de la playa con respecto a los trenes de oleaje. La parte norte de Playa Hermosa presenta excepcionalmente "beach cusps" frente a las otras, debido a que su posición Norte Sur bastante rectilinea permite que los trenes del oleaje océanico del SW. refractados paralelicen sus crestas fácilmente con ella, produciéndose en consecuencia vaivén. El sector de ubicación de los "beach cusps" en esta parte no debe ser de presencia muy banal, ya que se encuentra en fotos aéreas de años anteriores, en las que se aprecia incluso que más al sur en la bahía no hay "beach cusps" nítidos. Lo mismo se encontró en terreno. Hay que aducir en seguida que fue precisamente en Caleta Los Piures (extremo sur de Playa Hermosa) donde se observó en el campo la erosión y el retroceso más espectacular derivado de las marejadas de 1982, en una combinación entre vaivén y deriva. Todo muestra que en la parte media de la bahía que presenta más vaivén, la deriva es importante, como movimiento compensatorio de la erosión en la concavidad sur. Finalmente, se recuerda que el experimento de Silvester entregó transferencia por deriva desde el primero al segundo medio corazón.

\subsection{EL PROBLEMA DE LA ESTRUCTURA dE LOS SISTEMAS DE DUNAS}

Llama la atención que en dos bahias en zeta tan parecidas como Topocalma y Pichilemu, la estructura sea tan distinta. Es dificil relacionar este hecho con los rasgos morfométricos, salvo que estos sean cotejados con las condiciones de refracción del oleaje. Aun así, el fenómeno 
parece más bien una cuestión de balance de sedimentación. La cuenca fluvial relacionada con la playa de Topocalma tiene un área de más que el doble de la relacionada con la playa de Pichilemụ. La observación de terreno y en aerofoto revela mayor aporte de arenas en el primer caso, con una acción del viento muy efectiva. Las dunas longitudinales se forman a partir del lecho mismo del río antes de que éste desemboque. La playa, en cambio, abastece a los barjanes. Contrariamente, la pequeña cuenca de Pichilemu no parece entregar aportes muy significativos a la bahía, la que a su vez está más protegida con respecto al viento que Topocalma. Siendo la refracción del oleaje mayor que en ésta, el ataque a la máxima concavidad es más eficiente. Estos hechos coinciden con la mayor proporción de dunas parabólicas en Pichilemu.

Paralelamente, playa Cáhuil, con la orientación característica del estilo de playas sensiblemente rectas, está relacionada con la gran cuenca del Nilahue, de un orden de tamaño fuera de serie con respecto a las anteriores. Hay poca refracción del oleaje, pero buena exposición a éste y al viento. La poca refracción y la condición de playa poco oblicua y casi transversal, según la clasificación de Araya-Vergara (1982b), parece ser favorable a un equilibrio sedimentológico, sobre todo si se observa los perfiles de playa en el mapa de la Fig. 1. El carácter mixto de este sistema dunario puede ser debido a estos factores. A pesar del aparente desorden en este campo de dunas, hay cierta organización espacial discernible, porque las dunas primarias siempre están entre las más cercanas a la playa.

Por último, un análisis interesante consiste en determinar el grupo a que pertenecen las dunas del frente, las más alejadas de la playa. En Topocalma ellas son barjanes; en Pichilemu, parabólicas; y en Cáhuil, parabólicas y transversales.

\section{CONCLUSIONES}

6.1. En el intento de precisar las morfoestructuras fundamentales que tienen que ver con la irregularidad actual de la línea de costa, el sistema de correlaciones permite determinar un sistema morfológico en que los lineamientos tectónicos tienen claros efectos en los desalineamientos y curvaturas litorales.

6.2. Para señalar una hipótesis adecuada sobre la evolución general, el modelo de CLAPP parece correcto siguiendo el proceso de contraposición. Teniendo en cuenta la herencia del Cuaternario. la hipótesis ergódica permite confrontar diferencias actuales de estado explicadas por el fenómeno de la contraposición.

Si esta última está en relación con la epigénesis, la forma de una espiral logarítmica puede en parte ser heredada de un nivel del mar más antiguo, si esta forma planimétrica se repite en el interior de la bahía. El mismo proceso puede haberse repetido en niveles más altos que el actual (Schwartz y Gravert, 1970, en Yasso, 1982). Para Yasso, no se sabe si el ángulo espiral permanece constante a medida que el tamaño de la bahia crece con el tiempo.

6.3. La determinación de la influencia de estos factores en las características morfométricas de la línea litoral, desemboca no solamente en la expresión de un sistema morfológico, sino también en la de un sistema proceso-respuesta. En este sentido, los dos estilos diferentes pueden tratarse como subsistemas, en los que se encuentra diferentes respuestas en la morfogénesis actual. Si el ángulo de desalineamiento explica la mayor parte de los efectos, hay que recordar que él siempre resultó menor que $35^{\circ}$, lo que concuerda con lo generalizado por Davies. Éste se refiere a que la condición "offset" parece representar una condición entre transporte completamente libre y transporte completamente impedido, probablemente dependiente del ángulo. Lo analizado en el presente trabajo permite también asegurar una buena dependencia de la orientación con respecto a los trenes de olas significativas. Sólo en esta perspectiva puede argumentarse con Davies que la deriva litoral es limitada, ya que la forma de zeta es un ajuste a la refracción de las olas constructivas, pero que una estacionaria y lenta deriva unidireccional existe, transfiriendo sedimentos de una bahia a otra.

Ajustes computacionales recientes de espirales logarítmicas dan ángulos espirales distintos para bahías adyacentes a YaSso (1982), quien concluye que las causas de las diferencias no están aún comprendidas. Las correlaciones logradas en el presente trabajo permiten dar una primera respuesta aproximada a esta interrogante $y$ atribuir esas diferencias al grado de desalineamiento y a la orientación. Según esto, si el tamaño de la bahía crece con el tiempo, el ángulo espiral sólo debiera permanecer constante cuando la relación entre desalineamiento y orientación permanezca constante.

6.4. Señalando las posibles relaciones de estos hechos con el grado de estabilidad actual, debe 
llamarse la atención sobre nuevos fenómenos encontrados y su conducta en playas de líneas de costa contrapuestas y desalineadas. Si la refracción es una condición de equilibrio, la forma misma que se mantiene en equilibrio no puede ser claramente explicada sin la noción de umbral, ya que son las marejadas las que permiten una erosión notable explicando la mantención de la mayor curvatura. Por lo tanto, debe ser el equilibrio entre erosión y deposición el que explica la forma en zeta para diferentes desalineamientos. La comprensión de la sola refracción no basta sin determinar su modo de acción.

Muy recientes deducciones de Yasso (1982) concuerdan con los resultados del presente trabajo referente a la retrogradación de la orilla más curva de la espiral logarítmica por efecto de las olas refractadas.

6.5. Esto último permite trabajar con criterios más adecuados para la interpretación de los cambios. Las observaciones que se están realizando sobre avances, estabilidades o retrocesos de litorales arenosos pueden ser peligrosamente interpretadas si no se toma en cuenta los efectos de erosión y deposición diferencial para una misma playa. Las mediciones de KoIKE (1977) y OzASA (1977) en Japón muestran claramente diferencias de estado en una misma bahia, que llaman al estudio de factores no bien establecidos. Según el presente trabajo, parece ser que el mayor ángulo de "offset" tiende a aumentar las diferencias de estado, 0 , por lo menos, las diferencias de comportamiento frente a la mantención del equilibrio.

6.6. La teoria de las bahías y playas derivadas de desalineamiento puede ser por to tanto enriquecida estudiando el comportamiento diferencial necesariamente en el terreno, comparando situaciones de equilibrio temporal con resultados de umbrales, en un marco espacio-temporal.

6.7. Para ello es necesario mejorar la sistemática de los perfiles de playa, ya que la realidad de su revisión longitudinalmente en una bahía permite establecer tipos complejos, de cuya distribución depende la interpretación dinámica.

En un primer intento, la diferencia de estado en una misma playa se puede captar mejor si los perfiles son clasificados en erosionales y acrecionales, de acuerdo con el criterio del Coastal Enginnering Research Center (DAvIS, 1982), pudiéndose usar la categoría mixto para los casos complejos.

6.8. Desde el punto de vista del manejo del litoral, la dependencia de la dinámica diferencial en relación con el desalineamiento parece de substancial importancia. La construcción de rompeolas equivale a generar salientes artificiales en desalineamiento. Si hay abastecimiento natural de arenas por deriva, puede generarse una playa siguiendo el modelo de SILVESTER. Es exactamente lo que ocurrió en Constitución en la primera parte de este siglo cuando se construyó molos con el fin de generar un puerto marítimo. Muchos años después, en 1982, las continuas marejadas de invierno trataron diferencialmente la playa formada entre los molos. En la concavidad sur, la playa quedó con perfil cóncavo por lo menos hasta fines del verano de 1983 y delimitada por un microacantilado de unos $2 \mathrm{~m}$ en el terraplén de operaciones de la planta de celulosa, cuya estabilidad debe ser de aquí en adelante puesta en duda.

Por lo tanto, conociendo los atributos del sistema estructural, natural o artificial, que regula la forma de las playas y su evolución, es posible tener mejores antecedentes de su estado con fines de manejo.

6.9. De todo, se desprende que hay una notable dependencia sistémica entre el estilo morfoestructural, su grado de evolución, la condición de estado, la condición de ataque y el proceso de toma de decisiones, frente a los cambios.

La ubicación preferencial de las caletas y zonas de balnearios en las mayores curvaturas de las bahías en zeta, con demostraciones de perfiles de erosión después de las marejadas, es un hecho que merece atención en las estrategias de manejo que se basan en el sistema de recursos de la zona costera, como las que bosqueja HalLs (1982).

6.10. Hay que tener en cuenta que las observaciones de terreno en las playas fueron hechas después de un invierno excepcionalmente tormentoso en el Pacífico Sur. Esto aparentemente puede influir en el estado encontrado en la revisión, pero más en la intensidad de los fenómenos que en su significado mismo.

6.11. Finalmente, la distinción entre dunas primarias y secundarias parece un buen criterio para análisis de sistemas morfológicos. La orientación y el balance sedimentológico parecen ser los principales factores que permiten integrarlos a sistemas proceso-respuesta, pero sobre esto es necesario investigar más. 


\section{AGRADECIMIENTOS}

El autor agradece a los colegas Víctor Guillermo Quintanilla y Hugo Romero, del Departamento de Geografia, la revisión del manuscrito y sus útiles sugerencias. La Srta. Maria Angélica Mora puso en limpio prolijamente las ilustraciones. El trabajo de terreno y la adquisición de aerofotos fueron posibles gracias al apoyo del Departamento de Desarrollo de la Investigación de nuestra Universidad.

\section{REFERENCLAS}

Araya-Vergara, J.F. 1967. Morfometria de la curvatura de las playas entre Punta de Talca y Punta Toro (Chile Central). Inform. geogr. Chile (17): 5-30.

Araya-Vergara, J.F. 1971. Determinación preliminar de làs caracteristicas del oleaje en Chile Central. Mus. Nac Hist. Nat. Not. Mens. 15(174): 8-12.

Araya-Vergara, J,F, 1982a. Análisis de la localización de los procesos y formas predominantes de la línea litoral de Chile: observación preliminar. Inform. geogr. Chile (29): 35-55.

Araya-Vergara, J.F. 1982b. Beach orientation. In: Encyclopedia of Beaches and Coastal Environments. Hutchinson Ross Publish. Co. Strousdburg, Penn.

BIRD, E.C.F. 1981. Recent changes on the world's sandy shorelines. In: Brid, E.C.F. \& K. Koike, Eds. Coastal Dynamics and Scientific Sites. Intern. Geogr. Union Dep. of Geogr. Komazawa University, Tokio: 5-30.

Bruggen, J. 1950. Fundamentos de la geologia de Chile. Ed. I.G.M. Santiago.

Christofoletti, A. y A.G. Pires Neto. 1975. Morfometria planimétrica das praias entre Santos y Sao Sebastiao. R. Brasileria de Geogr. 37: 110-123.

Christofoletti, A. y A.G. Pires Neto. 1980. Estudio comparativo de las variables de la morfología planimétrica de las playas del iitoral paulista (Brasil). R. de Geogr. Barcelona. 14(1-2): 27-38.

CLAPp, H. 1983. Contraposed shorelines. J. of Geol. 21: 537 (En Johnson, 1919).

Darwin, Ch. 1906. Jeolojia de la América Meridional. Trad. Imp. Cervantes. Stgo

DAVIES, J.L. 1977. Geographical Variation in Coastal Development. Longman. London.

DAvis, R.A. JR. 1982. Beach profiles. In: Encyclopedia of Beaches and Coastal Environments. Hutschinson Ross Publ. Co. Stroudsburg. Pennsylvania: 159.

De Martonne, E. 1926. Traité de Géographie Physique. Tome Second: Le rélief du sol. 4 me. Ed. Colin. Paris. Dirección de Obras Portuarias y Tudor Eng. Co. 1968. Wave Study. Supplement. Constitución. Chile. Sn. Francisco. California.

Fuenzalida, H., R. Cooke, R. Paskoff, K. Segerstrom y W. Weischet. 1965. High stands of Quaternary Sea Level Along the Chilean Coast. Geol. Soc. of Amer. Inc. Special Paper 84: 473-496.

HAiLs, J.R. 1982. Coastal zone management. In: Encyclopedia of Beaches and Coastal Environments. Hutchinson Ross Publ. Co. Stroudsburg. Pennysilvania: 324-328.

Halugan, G.H. 1906. Sand movement on the New South Wales Coast. Proc. Linn. Soc. N.S.W. 31: 619-640 (En DAVIES, 1977).

Johnson, D.W. 1919. Shore Processes and Shoreline Development. Wiley. N.Y.

KING, C.A.M. 1972. Beaches and Coasts. Second Ed. Arnold. London.

KING, C.A.M. 1977. Classification and Morphometry of the Coast Between $20^{\circ} \mathrm{S}$. and $42^{\circ} \mathrm{S}$. R. Geogr. Valp. (8) 27-57.

KOIKE, K. 1977a. The Recent Change of Sandy Shorelines in Japan. Komazawa Geogr. (13): 1-16.

Kaumbein, W.C. 1947. Shore processes and beach characteristics. Tech. Memo. Beach. Eros. Bd. U.S. 3 (En DAVIES, 1977)

Ministefio de Obras Públicas y Dirección de Obras Portuarias, 1953-54. Rapport Général sur les travaux de la Mission d'Observations du Laboratoire Central d'Hydraulique de France: Au Port de San Antonio et á I'Embouchure de R. Maipo. $4^{3}$ Partie. Océanographie (Inédito).

OzaSA, H. 1977. Recent shoreline changes in Japan. Coastal Eng. in Japan. 20: 69-81.

SAKALOWSKY, P.P. 1975. Shore processes and changes in Napatree Beach, Rhode Island. Z. für Geomorph. N.F. 19(4): $460-478$

Silvester, R. 1960. Stabilization of sedimentary coastlines. Nature. 188(4749): 467-469.

Tudor ENG. Co. 1965. Report for a Waterfront Facility. Constitución, Chile. San Francisco. California.

YASSO W.E. 1965. Plan geometry of headland-bay beaches. J. Geol. 73: 702-714.

YASSO, W.E. 1982. Headland bay beach. In: Encyclopedia of Beaches and Coastal Environments. Hutchinson Ross Publ. Co. Stròdsburg. Pennsylvania: 460. 